Revista Arbitrada Interdisciplinaria KOINONIA

Año V. Vol V. N9. Enero - Junio 2020

Hecho el depósito de Ley: FA2016000010 ISSN: 2542-3088

FUNDACIÓN KOINONIA (F.K). Santa Ana de Coro. Venezuela.

Marco Iván Chávez-Cadena; Jaime Wilson Jiménez-Cargua; Mirian Isabel Cucuri-Pushug

http://dx.doi.org/10.35381/r.k.v5i9.647

\title{
Análisis de confiabilidad, mantenibilidad y disponibilidad (CMD) del sistema de reinyección de agua de formación
}

\section{Reliability, maintainability and availability (RMD) analysis of the training water reinjection system}

\author{
Marco Iván Chávez-Cadena \\ marco.chavezc@espoch.edu.ec \\ Escuela Superior Politécnica de Chimborazo, Sede Orellana \\ Ecuador \\ https://orcid.org/0000-0003-1204-3334 \\ Jaime Wilson Jiménez-Cargua \\ wilson.jimenez@espoch.edu.ec \\ Escuela Superior Politécnica de Chimborazo, Sede Orellana \\ Ecuador \\ https://orcid.org/0000-0003-0523-5805 \\ Mirian Isabel Cucuri-Pushug \\ mirian.cucuri@espoch.edu.ec \\ Escuela Superior Politécnica de Chimborazo, Sede Orellana \\ Ecuador \\ https://orcid.org/0000-0002-0141-4147
}

Recibido: 17 de noviembre de 2019

Revisado: 25 de noviembre de 2019

Aprobado: 28 de diciembre de 2019

Publicado: 20 de enero de 2020

\section{RESUMEN}

Con la finalidad de describir y analizar los aspectos fundamentales del sistema en estudio, se enmarco en cumplir con el proceso metodológico en función del objetivo general de la investigación: "Analizar la Confiabilidad, Mantenibilidad y Disponibilidad del sistema reinyección de agua de la empresa PETROAMAZONAS EP, Bloque 18 ZPF, mediante análisis CMD, el estudio fue conformada por cinco bombas multietapas las bombas P-1501A, P-1501B, P-1501C, P-1501D y P-1501E. Se calculó y analizo los índices de Mantenibilidad del sistema de reinyección de agua de la empresa PETROAMAZONAS. Los tiempos medios en las reparaciones MTTR, de las bombas P1501 A y P-1501 B se encuentran entre 30.98 y 23.67 horas; mientras que los MTTR de 
Revista Arbitrada Interdisciplinaria KOINONIA

Año V. Vol V. Nº. Enero - Junio 2020

Hecho el depósito de Ley: FA2016000010

ISSN: 2542-3088

FUNDACIÓN KOINONIA (F.K). Santa Ana de Coro. Venezuela.

Marco Iván Chávez-Cadena; Jaime Wilson Jiménez-Cargua; Mirian Isabel Cucuri-Pushug

para las bombas P-1501 C y P-1501 D están entre 3.66 y 12.79 horas. Finalmente, el MTTR de la bomba P-1501 E es de 100.76 horas.

Descriptores: Mantenimiento; ingeniería de la producción; tecnología de materiales; agua residual. (Palabras tomadas del Tesauro UNESCO).

\section{ABSTRACT}

With the purpose of describing and analyzing the fundamental aspects of the system under study, the methodological process was carried out in accordance with the general objective of the research: "To analyze the reliability, maintainability and availability of the water reinjection system of the PETROAMAZONAS EP company, Block 18 ZPF, by means of CMD analysis. The study was made up of five multistage pumps: P-1501A, P1501B, P-1501C, P-1501D and P-1501E. The maintainability indexes of the water reinjection system of the PETROAMAZONAS company were calculated and analyzed. The average times in MTTR repairs, of the pumps $\mathrm{P}-1501 \mathrm{~A}$ and $\mathrm{P}-1501 \mathrm{~B}$ are between 30.98 and 23.67 hours; while the MTTR for the pumps P-1501 C and P-1501 D are between 3.66 and 12.79 hours. Finally, the MTTR of the pump P-1501 E is 100.76 hours.

Descriptors: Maintenance; production engineering; materials technology; waste water. (Words taken from the UNESCO Thesaurus).

\section{INTRODUCCIÓN}

La investigación surge ante la necesidad de acciones que amenoren las pérdidas de producción suscitadas en base a los fallos que mantiene el sistema de reinyección de agua de Petroamazonas EP, Bloque 18. Si bien se han tomado acciones ante la problemática, hace falta un análisis de evaluación integral del proyecto de la gestión del mantenimiento del sistema de reinyección de agua, la presente investigación pretende ser ese medio que permita planear, ejecutar y controlar la gestión y operación del mantenimiento y brindar así propuestas que disminuyan las paradas innecesarias del sistema lo que implica menor cantidad de pérdidas de producción.

La Confiabilidad, Mantenibilidad y Disponibilidad son prácticamente las únicas medidas técnicas y científicas, fundamentadas en cálculos matemáticos, estadísticos y probabilísticos, que tiene el mantenimiento para su análisis y su evaluación integral y especifica; es así que el modelo universal de análisis de Confiabilidad, Mantenibilidad y 
Marco Iván Chávez-Cadena; Jaime Wilson Jiménez-Cargua; Mirian Isabel Cucuri-Pushug

Disponibilidad (CMD) propuesto para la presente investigación pretende aportar una metodología única y sintética que permita estimar los diferentes parámetros de una forma lógica, logrando así la comparación similar en el tiempo, ya sea con la propia industria o con otras.PETROAMAZONAS EP, es la empresa pública de exploración y explotación de hidrocarburos en el Ecuador. Dentro de sus activos se encuentra el Bloque 18, Palo Azul.

Durante el proceso de extracción de crudo es inevitable obtener agua de formación y gas. El campo Palo Azul produce a la fecha de investigación 18835 Barriles de petróleo por día (BPPD), junto con 60420 barriles de agua por día (BAPD) y 8481 miles de pies cúbicos de gas por día (MPCSD). El decreto ejecutivo 1215 del gobierno del Ecuador, indica que el agua de formación debe ser tratada y no puede descargarse a cuerpos de agua sin cumplir ciertos parámetros. El mismo decreto indica que se puede disponer de desechos líquidos por medio de reinyección en una formación porosa tradicionalmente no productora. Para el normal desempeño de las actividades del Bloque $18 \mathrm{y}$ con el creciente aumento de la cantidad de agua a reinyectar, se debe optimizar y asegurar cuan confiable es el sistema de reinyección actual.

En el año 2014 una de las bombas principales de reinyección de agua en Palo Azul, sufrió un modo de falla que conllevó a una reparación mayor. La falta de unidad de respaldo, obligó a buscar unidades de reinyección en otros campos de producción. La falta de mano de obra local calificada para reparar la bomba ha provocado que el tiempo de reparación sea sumamente elevado. Finalmente se colocó una bomba similar, mas no igual, este hecho produjo otro contexto operacional, otros modos de falla del sistema, y diferentes parámetros.

La problemática del sistema de reinyección ha ocasionado incluso paradas de pozos productores debido a la incapacidad de reinyectar el agua de formación precedente de los mismos. Desde Enero de 2015 hasta Junio de 2015, Palo Azul ha acumulado pérdidas totales por 17485.16 barriles de petróleo son 4037.0 horas acumuladas de pérdida. 
Marco Iván Chávez-Cadena; Jaime Wilson Jiménez-Cargua; Mirian Isabel Cucuri-Pushug

Es insostenible la cantidad de pérdidas de producción relacionadas a la falta de fiabilidad del sistema de reinyección de agua. Si siguieran las condiciones actuales (incrementando el corte de agua de los pozos productores y con el número de fallas e indisponibilidad de equipos) seguirán incrementándose proporcionalmente las pérdidas de producción. Es necesario contar con más equipos de respaldo entre otras alternativas que aseguren la capacidad de reinyección de agua en Palo Azul, sin dejar a un lado los datos de la proyección macro de la empresa PETROAMAZONAS EP en inversión para ir a la par de un análisis económico.

El análisis CMD brindará información necesaria para determinar el tiempo probable durante el cual el sistema esté en capacidad de cumplir su función (Disponibilidad), la probabilidad de que el sistema sea reparado a condiciones normales de operación dentro de un tiempo dado (Mantenibilidad) y la probabilidad de que el sistema desempeñe satisfactoriamente sus funciones por un espacio de tiempo dado y bajo un determinado conjunto de condiciones operativas (Confiabilidad). Así se formula la interrogante de investigación:

¿El análisis CMD permite realizar una evaluación integral del proyecto de la gestión del mantenimiento del sistema de reinyección de agua?

\section{MÉTODO}

Se abordó una investigación de tipo descriptiva con la finalidad de explicar y analizar aspectos fundamentales con la población de estudio, lo cual permitió cumplir con el proceso metodológico en función de alcanzar el objetivo general a planteado: "Analizar la Confiabilidad, Mantenibilidad y Disponibilidad del sistema reinyección de agua de la empresa PETROAMAZONAS EP, Bloque 18 ZPF, mediante análisis CMD".

La población estuvo constituida por el sistema de reinyección de agua del Bloque 18 de Petroamazonas, específicamente teniéndose como muestra el conjunto formado por las bombas multietapas, esto con el afán de personificar el sistema más crítico, ya que del mismo depende la operatividad del sistema. Este sistema está formado por cinco bombas multietapas las bombas P-1501A, P-1501B, P-1501C, P-1501D y P-1501E. 
Marco Iván Chávez-Cadena; Jaime Wilson Jiménez-Cargua; Mirian Isabel Cucuri-Pushug

Para la recogida de información se empleó lista de cotejo con la finalidad de recopilar información que luego sería suministrada al análisis de programas informáticos. Durante la investigación se utilizaron los softwares: RAM (Mora, 2007), FIASOFT, ICR v. 12.02.0.5 y SIM 1.7.

La línea de investigación del estudio propuesto corresponde al análisis y modelaje probabilístico de índices de Confiabilidad, Disponibilidad y Mantenibilidad. Para la medición CMD se utilizará el modelo universal e integral propuesto por Mora (2009) en su libro Mantenimiento EstratégicoEmpresarial:

Paso 1: Obtención de los datos de los tiempos útiles, fallas, reparaciones, tiempos perdidos de producción y mantenimiento, tiempos de suministros, demás tiempos requeridos. Se debe resaltar la importancia de la verosimilitud y correlación cronológica. Para el presente trabajo la base de datos MáximoOil\& Gas implantada en el año 2011 en el campo Palo Azul, PETROAMAZONAS EP, es la fuente de donde se obtuvo los tiempos de falla, tiempos de buen funcionamiento.

Paso 2: Trata sobre la preparación de los datos de fallas, reparaciones, tiempos útiles, mantenimientos, otras actividades, etc., dependiendo de la disponibilidad a usar (Genérica o de Steady-state, Inherente o Intrínseca, Alcanzada, Operacional y Operacional Generalizada) y de los requerimientos específicos del cálculo. Se separa lo correctivo de lo planeado. En la presente investigación la disponibilidad inherente es la que se calcula, esto por el hecho de no contar con tiempos exactos de demoras logísticas, suministros, retrasos. (Mora, 2007). Blanchard (1994) define a la disponibilidad inherente como la probabilidad de que el sistema opere satisfactoriamente cuando sea requerido en cualquier tiempo bajo las condiciones de operación especificadas y un entorno ideal de soporte logístico, es decir con la disponibilidad adecuada de personal, repuestos, herramientas, equipos de prueba y demás, sin considerar ninguna demora logística o administración. En los datos obtenidos de MaximoOil\& Gas existe la diferenciación entre tiempos de para del equipo sea por mantenimiento preventivo o por correctivo. Para el análisis de la disponibilidad inherente solo se utilizará los tiempos por mantenimientos correctivos. 
Marco Iván Chávez-Cadena; Jaime Wilson Jiménez-Cargua; Mirian Isabel Cucuri-Pushug

Paso 3: Decidir sobre el uso del Método de máxima verosimilitud o de Estimación de parámetros de No confiabilidad y de Mantenibilidad para obtener los valores más probables de los parámetros para una distribución dada. Para la presente investigación se utiliza el método ikaésimo tanto de forma manual como en la hoja de cálculo o software RAM adicional al libro Mantenimiento Estratégico Empresarial de Mora2007.

Paso 4: Realizar los cálculos de los parámetros de Weibull y validar las pruebas de bondad de ajuste, mediante tres pruebas Kolmogórov-Smirnov, Anderson-Darling y Chi cuadrado Ji2. Realizar la validación de bondad y ajuste para comprobar si los datos que se observan corresponden a la distribución escogida. Con la ayuda de la hoja de cálculo o software RAM adicional al libro Mantenimiento Estratégico Empresarial de Mora 2007, se verifica el cumplimiento de la función escogida. Con la ayuda de Excel y las formulas correspondientes se proceden a estimar los parámetros de $\beta, \eta$ y MTBF; estos parámetros calculados son comprobados con la hoja de cálculo o software RAM, Mora 2007 y también por el software FIASOFT, producto de la tesis "Desarrollo de un software para determinar los índices de fiabilidad en máquinas y equipos industriales" por Cristian Flores y Luis Hidalgo, ESPOCH 2008.

Paso 5: Parametrizar y realizar alineación o método de máxima verisimilitud que se requiere con otra función específica diferente a Weibull para estimar sus bondades de ajuste a partir del dato de Beta $(\beta)$ resultado del cálculo en el paso cuatro; definir cuál distribución aplica de acuerdo al parámetro. El objetivo de la alineación es estimar los parámetros de una línea recta que son el intercepto y la pendiente para minimizar el error (Mora, 2009).

Paso 6: Calcular los parámetros CMD acorde a la función seleccionada por el alineamiento. Analizar las curvas de densidad de falla, acumulada de fallas, confiabilidad y tasa de falla lambda. Realizar los pronósticos de curvas y parámetros. Con la ayuda de Excel y las formulas correspondientes se calcula el MTBF y MTTR para calcular la disponibilidad inherente de cada equipo. Las curvas resultado del análisis fueron obtenidas mediante el software RAM (Mora, 2007) y corroborados por FIASOFT. Finalmente se debe verificar el patrón y ajustar pronósticos CMD. Analizar 
Marco Iván Chávez-Cadena; Jaime Wilson Jiménez-Cargua; Mirian Isabel Cucuri-Pushug los parámetros en el tiempo. Definir una estrategia de mantenimiento acorde a los parámetros.

Cabe recalcar que una vez obtenidos los parámetros CMD de cada uno de los equipos analizados se conjugaron en un solo sistema, el sistema de reinyección de agua. Para conjugar estos parámetros se parte del hecho de que los equipos se encuentran en una estructura en paralelo o redundante activa. Esta conjunción a parte del cálculo en Excel realizado es corroborada por el "Software de Ingeniería de Confiabilidad y Riesgo ICR versión 12.02.0.5 desarrollado por CEANI, SIANI y Universidad de las Palmas de la GranCanaria.

\section{RESULTADOS}

\section{Análisis deestrategias}

Una vez obtenidos los índices de confiabilidad, mantenibilidad y disponibilidad de cada uno de los elementos y del sistema, se pueden analizar cada uno de sus parámetros para realizar ciertas conclusiones y aseveraciones. Las cinco bombas analizadas siguen una distribución de Weibull en sus diferentes etapas en lo que tiene que ver con la confiabilidad.

Para la bomba P-1501A y P-1501B que se encuentra en la etapa I de la fase III, la tasa de fallas se incrementa en varias formas causadas por fatiga, corrosión, envejecimiento, fricción y cargas cíclicas. Estas causas pueden ser disminuidas con tareas proactivas, reemplazo de componentes, tecnología,instrumentosavanzadosespecíficosenordentécnico,ejemplo:vigilanciade temperatura, control de corrosión, resistencia eléctrica, lubricación engrase y aceite, monitoreo de causas y efectos eléctricos, termografía infrarroja, análisis de vibraciones, ferrografía análisis de lubricantes, análisis espectométrico, cromatografía, líquidos penetrantes, ensayo de partículas magnéticas, ultrasonido, control de ruido, filtros magnéticos, corrientes inducidas. (Mora 2007).

Para la bomba P-1501C que se encuentra en la Fase II, rodaje o vida útil, con sus características: $\beta$ y Tasa de falla son fijos en valor, causados por: mantenimiento 
Marco Iván Chávez-Cadena; Jaime Wilson Jiménez-Cargua; Mirian Isabel Cucuri-Pushug inadecuado, entorno de trabajo, cargas aleatorias, errores humanos, situaciones fortuitas, eventos inesperados al azar. En esta fase puede mejorar sus parámetros CMD con rediseño de máquina o de proceso, revisión técnica de procesos u operaciones. (Mora 2007).

Para la bomba P-1501D y P-1501E que se encuentra en la Fase I, mortalidad infantil, tiene como característica la disminución paulatinamente o drásticamente de la tasa de falla con el tiempo. Sus causas de falla probables son: Defectos de producción, fallas de soldadura, fisuras, componentes imperfectos, calidad defectuosa, medio ambiente fuera de control, condiciones fuera de estándares, ensambles inadecuados, procesos inadecuados, se atenúan estas causa mediante chequeo de funcionamiento, control de calidad, examen de aceptación, FMECA o análisis de falla, acciones correctivas buenas. (Mora 2007).

A nivel táctico de mantenimiento, una vez claro la ubicación física de los equipos en sus curvas de falla, de tal forma que haya coherencia entre los estados de los equipos y la táctica que se desee implementar; La decisión de la táctica a seleccionar debe partir de la premisa de la fase que se encuentra en la curva de la bañera o Davies.

1. Para las bombas P-1501A y P-1501B cuyos $\beta$ están entre 1.05 y 1.95 la táctica recomendada es TPM y un poco deRCM.

2. Para la bomba P-1501C cuyo $\beta$ está entre 0.95 y 1.05 la táctica más adecuada es la Reactiva.

3. Para la bomba P-1501D y P-1501E cuyos $\beta$ están entre 0 y 0.95 la táctica recomendada aunque en menor medida que las otras es la Reactiva. 
Revista Arbitrada Interdisciplinaria KOINONIA

Año V. Vol V. N9. Enero - Junio 2020

Hecho el depósito de Ley: FA2016000010

ISSN: 2542-3088

FUNDACIÓN KOINONIA (F.K). Santa Ana de Coro. Venezuela.

Marco Iván Chávez-Cadena; Jaime Wilson Jiménez-Cargua; Mirian Isabel Cucuri-Pushug

\section{Cuadro 1}

Resultados de parámetros de confiabilidad de las bombas del sistema de reinyección de agua del ZPF

\begin{tabular}{ccccc} 
Bomba & $\begin{array}{c}\text { Parámetro } \\
\text { de forma } \\
\text { Beta }(\boldsymbol{\beta})\end{array}$ & $\begin{array}{c}\text { Parámetro } \\
\text { de escala } \\
(\boldsymbol{\eta})\end{array}$ & $\begin{array}{c}\text { Tiempo } \\
\text { medio entre } \\
\text { fallas } \\
\text { (MTBF) }\end{array}$ & $\begin{array}{l}\text { Distribución } \\
\text { que cumple u } \\
\text { Confiabilidad }\end{array}$ \\
\hline P-1501A & 1.37 & 1350.2 & 1234.51 & Weibull \\
P-1501B & 1.46 & 1504.9 & 1362.78 & Weibull \\
P-1501C & 0.995 & 670.23 & 671.81 & Weibull \\
P-1501D & 0.789 & 104.89 & 1197.31 & Weibull \\
P-1501E & 0.91 & 1287.51 & 1346.81 & Weibull
\end{tabular}

Fuente: Autor

Para la confiabilidad de cada una de las bombas del sistema de reinyección de agua, se obtuvo su ecuación, por cuanto la confiabilidad es un valor que depende del tiempo. Para la confiabilidad del sistema total de reinyección de agua se realizó un análisis del conjunto de bombas; las bombas P-1501A, P-1501B, P-1501C, P-1501D y P-1501E tienen una disposición enparalelo.

Mediante el software FIASOFT se comprobó los parámetros de la fiabilidad de cada una de las bombas. Como resultados también se obtuvo los gráficos de los índices de fiabilidad de cada una. Es así que la Fiabilidad, Desconfiabilidad, la densidad de la probabilidad de falla y la tasa de falla, se graficaron en función deltiempo 
Revista Arbitrada Interdisciplinaria KOINONIA

Año V. Vol V. №9. Enero - Junio 2020

Hecho el depósito de Ley: FA2016000010

ISSN: 2542-3088

FUNDACIÓN KOINONIA (F.K). Santa Ana de Coro. Venezuela.

Marco Iván Chávez-Cadena; Jaime Wilson Jiménez-Cargua; Mirian Isabel Cucuri-Pushug

\section{Cuadro 2}

Confiabilidad de las bombas y del sistema de reinyección de agua del ZPF

$$
\begin{array}{lllll}
\text { P-1501A P-1501B P-1501E P-1501C } & \text { P-1501D }
\end{array}
$$

$\begin{array}{llllll}\mathbf{R}(\mathbf{t}) & \mathbf{R}(\mathbf{t}) & \mathbf{R}(\mathbf{t}) & \mathbf{R}(\mathbf{t}) & \mathbf{R}(\mathbf{t}) & \text { CONFIABILIDAD } \\ \text { FUNCION DE } & \text { FUNCION DE } & \text { FUNCION DE } & \text { FUNCION DE } & \text { FUNCION DE } & \text { DEL SISTEMA } \\ \text { CONFIABILIDAD } & \text { CONFIABILIDAD } & \text { CONFIABILIDAD } & \text { CONFIABILIDAD } & \text { CONFIABILIDAD } & \end{array}$

\begin{tabular}{rrrrrrr}
\hline 0 & $100 \%$ & $100 \%$ & $100 \%$ & $100 \%$ & $100 \%$ & $100 \%$ \\
0,02 & $100 \%$ & $100 \%$ & $100 \%$ & $100 \%$ & $99,98 \%$ & $100 \%$ \\
0,04 & $100 \%$ & $100 \%$ & $99,99 \%$ & $99,99 \%$ & $99,97 \%$ & $100 \%$ \\
0,06 & $100 \%$ & $100 \%$ & $99,99 \%$ & $99,99 \%$ & $99,95 \%$ & $100 \%$ \\
0,08 & $100 \%$ & $100 \%$ & $99,99 \%$ & $99,99 \%$ & $99,94 \%$ & $100 \%$ \\
$199,9 \%$ & $100 \%$ & $99,85 \%$ & $99,85 \%$ & $99,59 \%$ & $100 \%$ \\
$599,9 \%$ & $99,97 \%$ & $99,36 \%$ & $99,24 \%$ & $98,53 \%$ & $100 \%$ \\
10 & $99,8 \%$ & $99,93 \%$ & $98,80 \%$ & $98,49 \%$ & $97,48 \%$ & $100 \%$ \\
15 & $99,7 \%$ & $99,88 \%$ & $98,28 \%$ & $97,74 \%$ & $96,55 \%$ & $100 \%$ \\
20 & $99,6 \%$ & $99,81 \%$ & $97,77 \%$ & $97,01 \%$ & $95,69 \%$ & $100 \%$ \\
25 & $99,5 \%$ & $99,74 \%$ & $97,27 \%$ & $96,28 \%$ & $94,88 \%$ & $100 \%$ \\
30 & $99,4 \%$ & $99,66 \%$ & $96,78 \%$ & $95,56 \%$ & $94,11 \%$ & $100 \%$ \\
35 & $99,3 \%$ & $99,58 \%$ & $96,31 \%$ & $94,84 \%$ & $93,38 \%$ & $100 \%$ \\
40 & $99,2 \%$ & $99,49 \%$ & $95,84 \%$ & $94,13 \%$ & $92,67 \%$ & $100 \%$ \\
\hline
\end{tabular}

Fuente: Autor

Para la bomba $\mathrm{P}-1501 \mathrm{~A}$ se obtuvo un parámetro de forma Beta, $\beta=1.373$, este parámetro indica que se encuentra en la etapa I de la fase III de la curva de Davies. Estas fallas se fundamentan en el desgaste de los elementos, envejecimiento o la perdida de funcionalidad, se generan por el tiempo, son causadas por el uso. La bomba se encuentra en la fase conocida como la de envejecimiento o desgaste. Este hecho se evidencia en los datos de la Tabla 1, donde se muestra que el cambio de sellos mecánicos fue una de las tareas que más impacto tuvo en los down time, esto acompañado por mejoras operativas de la unidad. También es de notar la gran 
Revista Arbitrada Interdisciplinaria KOINONIA

Año V. Vol V. N9. Enero - Junio 2020

Hecho el depósito de Ley: FA2016000010

ISSN: 2542-3088

FUNDACIÓN KOINONIA (F.K). Santa Ana de Coro. Venezuela.

Marco Iván Chávez-Cadena; Jaime Wilson Jiménez-Cargua; Mirian Isabel Cucuri-Pushug

cantidad de orden de trabajo para limpieza de strainer en succión, si bien se tiene dos strainers (uno respaldo del otro) han provocado muy bajas succiones hasta apagar labomba.

Para el parámetro de escala de la bomba P-1501A Eta, $\eta$ es el valor determinante para fijar la vida útil del componente; a sus 1350.2 horas se espera que falle con una seguridad del $63.2 \%$, la confiabilidad de la bomba a las 1350.2 horas es del $36.8 \%$, independientemente del valor de $\beta$.

Para la bomba P-1501A, el MTBF es de 1234.51 horas, este es el periodo recomendado para realizar mantenimiento preventivo. El período de tiempo entre mantenimientos no debe ir más allá de las 1234.51 horas entre mantenimientos preventivos, en ese instante la bomba tendrá una confiabilidad del $41.3 \%$, se espera que exista un $58.7 \%$ de posibilidades de falla. A las 1033.7 horas existe el $50 \%$ de posibilidades que falle o no falle la bomba P-1501A.

Para la bomba $\mathrm{P}-1501 \mathrm{~B}$ se obtuvo un parámetro de forma Beta, $\beta=1.448$, este parámetro indica que se encuentra en la etapa I de la fase III de la curva de Davies. Estas fallas se fundamentan en el desgaste de los elementos, envejecimiento o la perdida de funcionalidad, se generan por el tiempo, son causadas por el uso. La bomba se encuentra en la fase conocida como la de envejecimiento o desgaste. En el Anexo 02 se evidencia que las ordenes de trabajo de la bomba P-1501B tienen un alto down time por mejoras operativas; sin embargo si destaca el cambio de cojinetes, revisión de juego axial, corrección de liqueos y atascamientos de válvulas que son efectos del desgaste de elementos rotativos.

Para el parámetro de escala Eta de la bomba P-1501B, $\eta$ es el valor determinante para fijar la vida útil del componente; a sus 1522.81 horas se espera que falle con una seguridad del $63.2 \%$, es decir la confiabilidad de la bomba a las 1522.81 horas es del $36.8 \%$, independientemente del valor de $\beta$.

Para la bomba P-1501 B, el MTBF es de 1381.09 horas, este es el periodo recomendado para realizar mantenimiento preventivo. El período de tiempo no debe ir más allá de las 1381.09 horas entre mantenimientos preventivos, en ese instante la 
Revista Arbitrada Interdisciplinaria KOINONIA

Año V. Vol V. N9. Enero - Junio 2020

Hecho el depósito de Ley: FA2016000010

ISSN: 2542-3088

FUNDACIÓN KOINONIA (F.K). Santa Ana de Coro. Venezuela.

Marco Iván Chávez-Cadena; Jaime Wilson Jiménez-Cargua; Mirian Isabel Cucuri-Pushug bomba tendrá una confiabilidad del $41.9 \%$, se espera que un $58.1 \%$ de posibilidades de falla. A las 1182.5 horas existe el $50 \%$ de posibilidades que falle 0 no falle la bombaP-1501B.

Para la bomba $\mathrm{P}-1501 \mathrm{C}$ se obtuvo un parámetro de forma Beta, $\beta=0,995$, este parámetro indica que se encuentra en la fase II de la curva de Davies, de madurez o de vida útil. Las fallas se originan básicamente por operación indebida de los equipos, sobre carga de la capacidad de producción, cambios constantes en las condiciones de funcionamiento.

Para el parámetro de escala Eta de la bomba P-1501C, $\eta$ es el valor determinante para fijar la vida útil del componente; a sus 670.23 horas se espera que falle con una seguridad del $63.2 \%$, es decir la confiabilidad de la bomba a las 670.23 horas es del $36.8 \%$, independientemente del valor de $\beta$. Para la bomba P-1501 C, el tiempo medio entre fallas es de 671.81 horas, este es el periodo recomendado para realizar mantenimiento preventivo. Es decir mediante los cálculos anteriores se puede indicar que el período de tiempo no debe ir más allá de las 671.81 horas entre mantenimientos preventivos, en ese instante la bomba tendrá una confiabilidad del $36.7 \%$, seespera que un $63.3 \%$ de posibilidades de falla. A las 463 horas existe el $50 \%$ de posibilidades que falle o no falle la bombaP-1501C.

Para la bomba P-1501D se obtuvo un parámetro de forma Beta, $\beta=0,804$, este parámetro indica que se encuentra en la fase I de la curva de Davies, de rodaje o de mortalidad infantil. Las fallas se deben normalmente a defectos de materiales, diseños inadecuados, montajes deficientes, mantenimientos incorrectos, calidad deficiente en elementos y repuestos.

Para el parámetro de escala Eta de la bomba P-1501D, $\eta$ es el valor determinante para fijar la vida útil del componente; a sus 1016.74 horas se espera que falle con una seguridad del $63.2 \%$, es decir la confiabilidad de la bomba a las 1016.74 horas es del $36.8 \%$, independientemente del valor de $\beta$.

Para la bomba P-1501 D, el MTBF es de 1147.2 horas, este es el periodo recomendado para realizar mantenimiento preventivo. El período de tiempo no debe ir 
Marco Iván Chávez-Cadena; Jaime Wilson Jiménez-Cargua; Mirian Isabel Cucuri-Pushug más allá de las 1147.2 horas entre mantenimientos preventivos, en ese instante la bomba tendrá una confiabilidad del $34.11 \%$, se espera que un $65.89 \%$ de posibilidades de falla. A las 657 horas existe el $50 \%$ de posibilidades que falle o no falle la bomba P-1501D.

Para la bomba P-1501E se obtuvo un parámetro de forma Beta, $\beta=0,91$, este parámetro indica que se encuentra en la fase I de la curva de Davies, de rodaje o de mortalidad infantil. Las fallas se deben normalmente a defectos de materiales, diseños inadecuados, montajes deficientes, mantenimientos incorrectos, calidad deficiente en elementos y repuestos. Es evidente que el mayor problema de la bomba P-1501E fue producto del mal diseño de su tecnología de ajuste (rotura de cuerpo) provocó un down time elevado acompañado de problemas de alineación posteriores. Su montaje fue deficiente y encontrar la modificación adecuada conllevo tiempo y ensayos de prueba y error.

Para el parámetro de escala Eta de la bomba P-1501E, $\eta$ es el valor determinante para fijar la vida útil del componente; a sus 1287.51 horas se espera que falle con una seguridad del $63.2 \%$, es decir la confiabilidad de la bomba a las 1287.51 horas es del $36.8 \%$, independientemente del valor de $\beta$.

Para la bomba P-1501 E, el tiempo medio entre fallas es de 1346.81 horas, este es el periodo recomendado para realizar mantenimiento preventivo. Es decir mediante los cálculos anteriores se puede indicar que el período de tiempo no debe ir más allá de las 1346.81 horas entre mantenimientos preventivos, en ese instante la bomba tendrá una confiabilidad del $35.28 \%$, se espera que un $64,72 \%$ de posibilidades de falla. A las 861 horas existe el $50 \%$ de posibilidades que falle o no falle la bomba P-1501E. 
Revista Arbitrada Interdisciplinaria KOINONIA

Año V. Vol V. N9. Enero - Junio 2020

Hecho el depósito de Ley: FA2016000010

ISSN: 2542-3088

FUNDACIÓN KOINONIA (F.K). Santa Ana de Coro. Venezuela.

Marco Iván Chávez-Cadena; Jaime Wilson Jiménez-Cargua; Mirian Isabel Cucuri-Pushug

\section{Cuadro 3}

Parámetros de mantenibilidad de las bombas del sistema de reinyección de agua del ZPF.

\begin{tabular}{ccccc} 
Bomba & $\begin{array}{c}\text { Parámetro de } \\
\text { forma Beta }(\boldsymbol{\beta})\end{array}$ & $\begin{array}{c}\text { Parámetro de } \\
\text { escala }(\mathbf{n})\end{array}$ & $\begin{array}{c}\text { Tiempo medio } \\
\text { entre fallas } \\
\text { (MTBF) }\end{array}$ & $\begin{array}{l}\text { Distribución que } \\
\text { cumple } \\
\text { u Confiabilidad }\end{array}$ \\
\hline P-1501A & 0.668 & 22.606 & 29.95 & Weibull \\
P-1501B & 1.679 & 1.7 & 23.67 & LogNormal \\
P-1501C & 1.014 & 0.8 & 3.66 & LogNormal \\
P-1501D & 1.785 & 1.2 & 12.79 & LogNormal \\
P-1501E & 0.5735 & 59.32 & 94.96 & Weibull
\end{tabular}

Fuente: Autor

Para la bomba P-1501A toma un promedio de 29.95 horas salir de su acciones correctivas (MTTR), Siendo las tareas de modificación o de mejoras las que más tiempo tomaron (Implementación del sistema de alivio de presión, inserción de nuevo sistema de sellos y actualización de software de HMI).

Para la segunda bomba P-1501B toma un promedio de 14.2 horas superar las tareas correctivas y poner a punto el sistema. Las tareas de mejora de sellos fueron las que más tiempo dejaron inhabilitada la bomba, adicional en este activo se tuvo un cambio de cojinetes que contribuye a su MTTR. Se debe recalcar que el menor promedio de down time puede deberse a las lecciones aprendidas con la primera unidad y aprovechar los tiempos generados por otras fallas para realizar tareas como actualización de software, disminuyendo así su down time).

Para las bombas P-1501C y D se tienen MTTR similares de 3.4 y 5.7 horas, esto evidencia una rápida actuación del equipo de MNT y la relativa facilidad de mantenimientos de las bombas HPS Reda, Su fácil intercambiabilidad de piezas y elementos que requieren poco mantenimiento preventivo hacen de la tecnología de Schlumberger la más fácil de mantener, sin embargo su capacidad de reinyección está enfocada en términos de mediana cantidad, característica que las bombas $\mathrm{P}$ - 
Marco Iván Chávez-Cadena; Jaime Wilson Jiménez-Cargua; Mirian Isabel Cucuri-Pushug 1501 A y P-1501B marca Sulzer cumplen de mejor manera, ya que doblan y hasta triplica en su capacidad de reinyección de barriles de agua pordía.

Finalmente la bomba P-1501E HPS marca General Electric tiene el más alto MTTR, toma un promedio de 94.96 horas superar las fallas suscitadas. Esto en parte se debe a su diseño realizado en campo básicamente (proyecto donde se utilizó cuerpos de bomba, cámara de empuje y motor eléctrico que se disponía en ese instante y no con una ingeniería original del fabricante). Adicional, las fallas en sistemas auxiliares (sistema de seguridad e instrumentación) han tomado más tiempo del normal, las continuas mejoras o modificaciones de sus parámetros tanto por parte de la operadora como del fabricante han hecho un verdadero rompecabezas sumantenibilidad.

\section{Cuadro 4}

Resultados de la disponibilidad de las bombas de reinyección y el sistema total de reinyección.

Bomba Disponibilidad

\begin{tabular}{lc} 
P-1501 ${ }^{\text {a }}$ & $97.55 \%$ \\
P-1501B & $98.31 \%$ \\
P-1501C & $99.49 \%$ \\
P-1501D & $98.94 \%$ \\
P-1501E & $93.04 \%$ \\
Sistema de reinyección de agua & $\mathbf{8 3 . 8 \%}$ \\
\hline
\end{tabular}

Fuente: Autor

Como se puede observar se tiene una disponibilidad del sistema de reinyección de agua 0.838. La disponibilidad del $83 \%$ no cumple el indicador deseado para Petroamazonas EP, la empresa tiene una meta de disponibilidad de equipos más alta como indicador clave de mantenimiento (KPI) por lo que implícitamente se debe mejorar la confiabilidad delsistema. 
Revista Arbitrada Interdisciplinaria KOINONIA

Año V. Vol V. N9. Enero - Junio 2020

Hecho el depósito de Ley: FA2016000010

ISSN: 2542-3088

FUNDACIÓN KOINONIA (F.K). Santa Ana de Coro. Venezuela.

Marco Iván Chávez-Cadena; Jaime Wilson Jiménez-Cargua; Mirian Isabel Cucuri-Pushug

\section{CONCLUSIONES}

Se determinó los índices de Confiabilidad del sistema de reinyección de agua de la empresa PETROAMAZONAS EP, Bloque $18 \mathrm{ZPF}$, en base a los tiempos de operación o buen funcionamiento de las bombas principales P-1501A, P-1501B, P1501C, P-1501D yP-1501E.

Se determinó la curva de fiabilidad del sistema de reinyección de agua de la empresa PETROAMAZONAS EP, Bloque 18 ZPF, esto es la conjunción en paralelo de las bombas principales P-1501A, P-1501B, P-1501C, P-1501D yP-1501E.

Las bombas principales P-1501A, P-1501B, P-1501C, P-1501D y P-1501E son las que dominaron los datos del sistema de reinyección, el resto de equipos del sistema: bombas auxiliares, tanques y tuberías, no aportaron mayormente al histórico de fallas, de aquí la premisa que las bombas principales son las que simbolizan al sistema de reinyección de agua de Petroamazonas B18 ZPF, esto permite manifestar que el presente análisis CMD identifica plenamente al sistema deestudio.

Según la curva de fiabilidad del sistema de reinyección de agua calculada existe un periodo de alrededor de 42 días donde el sistema de reinyección de agua de la empresa PETROAMAZONAS EP, Bloque 18 ZPF, se mantendrá 100\% disponible; después de este período es muy probable encontrar una falla en el sistema que inhabilite la función principal del mismo.

Las bombas P-1501 A, B. D y E mantuvieron un tiempo medio entre fallas MTBF, similar de 1289 horas en promedio. La excepción de este hecho la mantuvo la bomba P-1501C que mantuvo un MTBF de 671.81 horas, por lo que claramente requiere serintervenida.

Las bombas principales del sistema de reinyección de agua mantuvieron un comportamiento de similitud en sus parámetros de forma $\beta$, por sus marcas y tiempo de estadía en el sistema. Es así que las bombas P-1501A y P-1501B marca Sulzer mantienen un $\beta$ promedio de 1.41 , siendo las primeras bombas instaladas y por ende las más utilizadas. Las bombas P-1501C y P-1501D marca Schlumberger mantienen un $\beta$ promedio de 0.89 siendo relativamente recién instaladas y se encuentran el 
Marco Iván Chávez-Cadena; Jaime Wilson Jiménez-Cargua; Mirian Isabel Cucuri-Pushug periodo de mortalidad infantil. Finalmente la bomba P-1501E marca General Electric mantiene un $\beta$ de 0.91 , esta fue la última bomba instalada en el sistema y tuvo algunas actualizaciones o modificaciones de diseño debido a su mortalidad infantil, sin embargo aún mantiene el status en la curva de Davies o de laBañera.

Se calculó y analizo los índices de Mantenibilidad del sistema de reinyección de agua de la empresa PETROAMAZONAS EP, Bloque $18 \mathrm{ZPF}$, en base a los tiempos de reparación de las bombas principales P-1501A, P-1501B, P-1501C, P-1501D yP$1501 \mathrm{E}$.

Los tiempos medios entre reparaciones MTTR, de las bombas P-1501 A y P-1501 B se encuentran en 30.98 y 23.67 horas. Mientras que los MTTR de 3.66 y 12.79 horas para las bombas P-1501 C y P-1501 D. Finalmente el MTTR de la bomba P-1501 E es de 100.76 horas. Los indicadores anteriores manifiestan que el sistema de reinyección de agua debe tener una autonomía (capacidad de almacenamiento de agua de reinyección) mínima de 4 días sin una de susbombas.

Mediante simulación Montecarlo se pudo pronosticar la disponibilidad del sistema de reinyección de agua, su indicador de 0.83 para 8760 horas, fue obtenido en promedio de 400 repeticiones desimulación.

\section{FINANCIAMIENTO}

No monetarios

\section{AGRADECIMIENTO}

A la Escuela Superior Politécnica de Chimborazo, Sede Orellana, por el apoyo técnico, científico realizado a la presente investigación. 
Revista Arbitrada Interdisciplinaria KOINONIA

Año V. Vol V. №9. Enero - Junio 2020

Hecho el depósito de Ley: FA2016000010

ISSN: 2542-3088

FUNDACIÓN KOINONIA (F.K). Santa Ana de Coro. Venezuela.

Marco Iván Chávez-Cadena; Jaime Wilson Jiménez-Cargua; Mirian Isabel Cucuri-Pushug

\section{REFERENCIAS CONSULTADAS}

Análisis RAM (Reliability, Availability, Maintainability) Chilworth a Dekra company, Valencia-Zaragoza-Barcelona-Madrid. [RAM Analysis (Reliability, Availability, Maintainability) Chilworth a Dekra company, Valencia-Zaragoza-BarcelonaMadrid]. Recuperado de: http://www.chilworth.es/register.php

Caña Puche, A. (2006). Análisis RAM de la planta de inyección de agua Resor de petróleos de Venezuela, S.A. (Tesis inédita de especialidad, Universidad Simón Bolívar). [RAM analysis of the Resor de Petróleos de Venezuela, S.A. water injection plant (Unpublished thesis of specialty, Simón Bolívar University)]. Recuperadode: https://n9.cl/bn1u

Decreto Ejecutivo 1215, Registro Oficial 265 de 13 de Febrero del 2001. Reglamento ambiental para las operaciones hidrocarburíferas del Ecuador, 2001. [Executive Decree1215, Official Registry 265 of February 13, 2001. Environmental regulations for hydrocarbon operations in Ecuador, 2001]. Quito, Ecuador.

Minitab (2016). Distribución De Weibull. [Weibull Distribution]. Recuperado de https://n9.cl/wpgh

Gómez de la Vega, H, Medina, N. Semeco, K \& Yánez, M. Análisis de la confiabilidad, Disponibilidad y Mantenibilidad en Sistemas Productivos. [Analysis of reliability, availability and maintainability in production systems]. Recuperado de: https://n9.cl/sg7h

Guevara, F., Kondo, N., Souza, G. y Patiño C. (2007). Método para evaluación de disponibilidad en sistemas de generación de energía eléctrica- aplicado a turbinas de gas -2016. [Method for evaluating availability in electric power generation systems - applied to gas turbines - 2016].Recuperado de: https://n9.cl/2q5to

Hernando Gómez de la Vega, Medina N, Semeco K, Yanez M. Análisis de la confiabilidad, Disponibilidad y Mantenibilidad en Sistemas Productivos. [Analysis of reliability, availability and maintainability in production systems]. Recuperado de: https://n9.cl/sg7h

Mora Gutierrez, A., (2007) Mantenimiento Estratégico Empresarial, Enfoque sistemático Kantiano, Medellín, Colombia: Fondo Editorial Universidad EAFIT. [Strategic Business Maintenance, Kantian Systematic Approach, Medellín, Colombia: Editorial Fund EAFIT University]. 
Revista Arbitrada Interdisciplinaria KOINONIA

Año V. Vol V. №9. Enero - Junio 2020

Hecho el depósito de Ley: FA2016000010

ISSN: 2542-3088

FUNDACIÓN KOINONIA (F.K). Santa Ana de Coro. Venezuela.

Marco Iván Chávez-Cadena; Jaime Wilson Jiménez-Cargua; Mirian Isabel Cucuri-Pushug

Placencia Urrutia, S. (2015) Módulo XII: Gestión Tecnológica Y Económica de Activos Físicos, (Maestría en Gestión del Mantenimiento, Escuela superior Politécnica de Chimborazo). [Module XII: Technological and Economic Management of Physical Assets, (Master in Maintenance Management, Higher Polytechnic School of Chimborazo)].

Rivera Mejía, J. Unidad I.- Conceptos Básicos de Confiabilidad. [Basic Concepts of Reliability]. Recuperado de: https://n9.cl/olkum

Sexto, L.F. (2015) Módulo: Ingeniería de la fiabilidad. Maestría en Gestión del Mantenimiento, Escuela superior Politécnica de Chimborazo, Ecuador. [Module: Reliability Engineering. Master in Maintenance Management, Higher Polytechnic School of Chimborazo, Ecuador].

Vergara Rea, E.J. (2007). Análisis de confiabilidad, Disponibilidad y Manteneabilidad del sistema de crudo diluido de Petrozuata, (Tesis inédita de Especialización, Universidad Simón Bolívar). [Reliability, Availability and Maintainability Analysis of the Petrozuata diluted crude system, (Unpublished Thesis of Specialization, Simón Bolívar University)]

Yanchapaxi Novillo, E. (2010) Estudio del sistema de reinyección de agua de formación del EPF (facilidades de producción edén) y diseño de una solución que permita optimizar la presión y el caudal, así como mejorar el control y operación del sistema (Tesis de Grado, Escuela Superior Politécnica del Ejercito). [Study of the reinjection system of EPF formation water (Eden production facilities) and design of a solution that allows optimizing pressure and flow, as well as improving the control and operation of the system (Bachelor Thesis, Higher Polytechnic School of the Army)]. Recuperado de:http://repositorio.espe.edu.ec/handle/21000/2806 